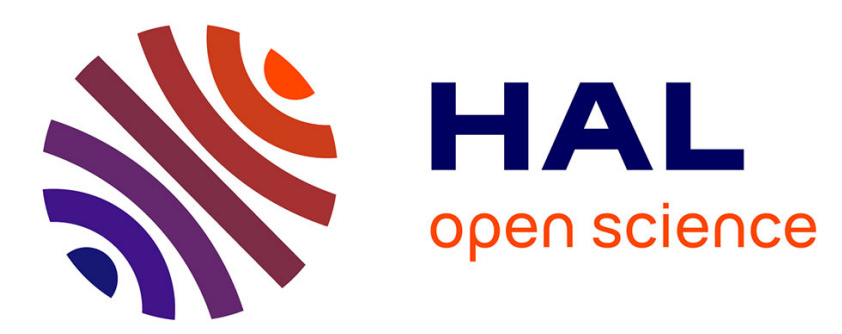

\title{
The study of displacement of immiscible fluids in porous media with constant pressure drop by means of nuclear tracers
}

\author{
A. Calvo, R. Chertcoff, M. Rosen, E. Guyon
}

\section{- To cite this version:}

A. Calvo, R. Chertcoff, M. Rosen, E. Guyon. The study of displacement of immiscible fluids in porous media with constant pressure drop by means of nuclear tracers. Revue de Physique Appliquée, 1989, 24 (5), pp.553-561. 10.1051/rphysap:01989002405055300 • jpa-00246081

HAL Id: jpa-00246081

https://hal.science/jpa-00246081

Submitted on 1 Jan 1989

HAL is a multi-disciplinary open access archive for the deposit and dissemination of scientific research documents, whether they are published or not. The documents may come from teaching and research institutions in France or abroad, or from public or private research centers.
L'archive ouverte pluridisciplinaire HAL, est destinée au dépôt et à la diffusion de documents scientifiques de niveau recherche, publiés ou non, émanant des établissements d'enseignement et de recherche français ou étrangers, des laboratoires publics ou privés. 


\title{
The study of displacement of immiscible fluids in porous media with constant pressure drop by means of nuclear tracers
}

\author{
A. Calvo $\left({ }^{1}\right)$, R. Chertcoff $\left({ }^{1}\right)$, M. Rosen $\left({ }^{1}\right)$ and E. Guyon $\left({ }^{2}\right)$ \\ (1) Departamento de Fisica, Facultad de Ingenieria, Universidad de Buenos Aires, Paseo Colón 850, \\ 1063 Buenos Aires, Argentina \\ (2) Laboratoire d'Hydrodynamique et de Mécanique Physique, ESPCI, UA CNRS 857, 10 rue Vauquelin, \\ 75231 Paris Cedex 05, France
}

(Reçu le 11 octobre 1988, révisé le 3 janvier 1989, accepté le 27 janvier 1989)

\begin{abstract}
Résumé. - Nous présentons des mesures de déplacement de fluides immiscibles dans les milieux poreux réalisées à l'aide de traceurs radioactifs. Nous avons injecté de l'eau marquée avec ${ }^{131}$ I dans ce milieu, d'abord rempli de kérosène (qui est ici le fluide le moins mouillant pour le poreux). On obtient les profils de saturation en mesurant la variation de l'activité nucléaire en fonction du temps et de la distance suivant l'écoulement. Nous avons appliqué cette technique à une première et à une seconde imbibition séparées par une phase de drainage. Nous avons utilisé comme milieux poreux des empilements non consolidés de billes de verre et des échantillons naturels de grès de Berea. On obtient des résultats similaires dans les deux cas. Nous comparons les résultats à des modèles théoriques. Une analyse simple fait apparaître le rôle dominant des effets capillaires dans nos expériences réalisées à basse vitesse avec un écoulement induit par une chute de pression faible et constante.
\end{abstract}

\begin{abstract}
A laboratory technique based on the use of nuclear tracers has been developed for studying the displacement of immiscible fluids in a porous medium. Water tagged with ${ }^{131} \mathrm{I}$ is injected in a porous medium initially filled with kerosene (a non wetting fluid for the medium). The saturation profiles are obtained by recording the nuclear activity as a function of position and time. The technique is applied to first and second imbibition separated by a phase of drainage. The porous media are non consolidated packings of microspheres and Berea sandstones. Similar results are obtained in both cases. The results are compared with theorical models (such as that of hyperdiffusion introduced by de Gennes). We present a simple analysis emphasizing the dominant role of capillary effects in the slow invasion experiments performed by us under a constant pressure head; it is shown to explain qualitatively the results.
\end{abstract}

\section{Introduction.}

Immiscible two phase flow in a porous medium has been intensively studied because of its applications to hydrology, chemical engineering and secondary oil recovery. The displacement of one phase by a second one is called drainage if the displaced fluid wets the porous medium, imbibition in the opposite case.

The physics of the displacement involves a complex interplay between the convective motion, the effect of viscosity and that of the presence of interfaces between fluids in contact with the medium [1].

A series of review articles by Anderson [2] presents numerous works on the effects of wetting properties on two phase flows in porous media.

One of the key problems is to determine whether the motion of the fluids in the material follows classical laws such as described by BuckleyLeverett [3], or displays anomalous features such as suggested by de Gennes [4]. Another important aspect is the dependence of the flow structure on the invasion process used.

The wetting properties of the fluid initially saturating the medium are also particulary relevant.

In the present work, in order to analyse these problems, we take advantage of the flexibility of the radiotracer technique : it allows, during the experiment, in-situ measurements of fluid saturations as a function of time and distance along the flow.

In addition, we have used a constant driving pressure technique to induce the flow : this is closer to slow oil recovery conditions. The role of the precursory film flow of the wetting phase can be essential. This has been shown in particular in model 
experiments by Lenormand [5], and in the theoretical work of de Gennes [6]. Therefore, we have attempted to eliminate the influence of these wetting fluid films on the pore walls. For that purpose, the medium is initially saturated by the less wetting fluid (kerosene) of the water (w)-kerosene (o) couple we have used.

Let us now describe in more detail the actual experimental procedure which we have used and the relative values of the physical parameters controlling the process.

\section{Experimental procedure.}

2.1 RELATIVE MAGNITUDE OF THE PHYSICAL PARAMETERS IN OUR EXPERIMENT. - Gravitational forces can affect strongly the profiles of the fronts if fluids of different densities are used ; this is particularly the case with samples elongated in the vertical direction. This last effect can be estimated from the dimensionless Bond number: it is weak in the conditions of the present experimental work and will be neglected. More precisely, the Bond number can be defined as $\Delta \rho g a^{2} / \mu$ where $\Delta \rho=\rho_{\mathrm{w}}-\rho_{\mathrm{o}}$ is the density difference between the two phases $\left(\rho_{\mathrm{w}}=\right.$ $\left.1 \mathrm{~g} / \mathrm{cm}^{3}, \rho_{\mathrm{o}}=0.8 \mathrm{~g} / \mathrm{cm}^{3}\right)$. The present experiment uses a long horizontal cylindrical sample and the flow is axial (and therefore horizontal). Bo is of the order of $10^{-7}$. This suggests that there is no dependence of the saturation profile on the vertical distance.

Similarly, the balance between capillary and viscous forces is expressed through the capillary number $\mathrm{Ca}$; one has $\mathrm{Ca}=\mu_{\mathrm{w}} v / \gamma$ where $\mu_{\mathrm{w}}$ is the wetting fluid viscosity, $v$ the velocity and $\gamma$ the surface tension between the fluids. As noted by Wilkinson [7], additional length ratios such as channel length over radius or penetration length over radius must be taken into account. These length ratios behave as large multiplicative prefactors to the capillary number $\mathrm{Ca}$. One should therefore be careful of not neglecting too rapidly the effect of the viscosity even at low values of $\mathrm{Ca}$. (Similar corrections have to be applied to the Bond number in order to neglect the influence of gravity forces.)

In the present work, we characterize $\mathrm{Ca}$ by using the maximum flow rate value during a given experimental run. The viscosity and the density of water (w) and kerosene (o) are of the same order $\left(\mu_{\mathrm{w}} \sim 1 \mathrm{cp}, \mu_{\mathrm{o}} \sim 1.4 \mathrm{cp} ; \rho_{\mathrm{w}}=1, \rho_{\mathrm{o}}=0.75\right)$, and the surface tension between them is of the order of $30 \times 10^{-3} \mathrm{~N} / \mathrm{m}$. Typical values of $\mathrm{Ca}=10^{-7}$ to $10^{-6}$ are found.

Theoretically, in the limit of zero Bond and capillary numbers, drainage has been shown to be equivalent to a percolation process [8].

On the other hand, the influence of finite non zero values for $\mathrm{Bo}$ and $\mathrm{Ca}$ on a drainage invasion has been considered in several experimental and theoretical works : a satisfactory description of the geometry and properties of this process is at hand [7, 8]. However the situation is rather more complex and subtle in the case of imbibition.

2.2 CyCles OF INJECTIONS. - We have used the following sequence of injections :

- the medium is initially fully saturated with kerosene ;

- during a first imbibition, the wetting phase (tagged water) displaces the non-wetting kerosene. Let us emphasize that we speak of imbibition by reference to water injection since we cannot perform directly a measurement of the contact angles. Although it is known that variable wettability problems may be important in some materials [9], all our experimental results were coherent with the hypothesis of an imbibition process;

- during a drainage phase, water is expelled leaving only irreducible water at a saturation level $S_{\text {wir }}: S_{\text {wir }}$ is a function of the conditions of injection. The saturation ratio, $S(0<S<1)$, represents the ratio of the volume of one fluid phase and the total volume ; this ratio is estimated by averaging over a volume of the porous medium small enough compared to the extension of the front ;

- in the second imbibition, we inject water again. When new water is present at the same time as the residual one, the present technique allows to differentiate between them : we obtain this result by using ordinary water in the first step and tagged water in the second one. This is an important information since we can expect the residual water left after the first imbibition to modify the conditions of wettability of the porous medium [10].

2.3 Porous MATERIAL. - Two types of porous media are used: beds of glass microspheres and cylinders of Berea sandstone. The latter were calcinated at $1000{ }^{\circ} \mathrm{C}$ to stabilize their clay deposits [11].

The characteristics of the porous media are shown in table I. The porosity is computed by measuring the increase of weight between the dry medium and

Table I. - Porous media characteristics.

\begin{tabular}{|l|c|c|}
\hline & $\begin{array}{c}\text { Glass } \\
\text { microspheres }\end{array}$ & $\begin{array}{c}\text { Berea } \\
\text { sandstone }\end{array}$ \\
\hline Particle diameter $(\mu \mathrm{m})$ & $420-210$ & $\sim 2.5$ \\
Length $(\mathrm{cm})$ & 39 & 30 \\
Diameter $(\mathrm{cm})$ & 4 & 5 \\
Porosity (\%) & 34 & 22 \\
Permeability (Darcy) & 36.5 & 1.3 \\
\hline
\end{tabular}


the medium totally saturated by kerosene using a vacuum technique.

The Darcy permeability is obtained from the volume of fluid exiting from the porous medium per unit time for a given applied pressure difference. The outlet is at atmospheric pressure and the inlet is connected to a large vessel located above the level of the porous sample. In all experiments, the axis of the porous medium is horizontal. An example of such recordings is given for microspheres and for Berea sandstone in figure 1.
2.4 NUCLEAR TECHNIQUE. - The nuclear technique we have used has been described in a previous paper [12]. Let us recall briefly its main aspects : a nuclear tracer, ${ }^{131} \mathrm{I}$, of half lifetime 8 days, is added to water as $\mathrm{NaI}$ salt at a low dilution (typically 0.1 $\mathrm{ml} / \mathrm{l}$ ). This tracer has been chosen because of its low solubility in the non-wetting kerosene phase. The activity is detected using a scintillator counter. We record the variation of activity in planes perpendicular to the axis of the cylinder : we use a collimating system with an axial resolution of $3 \mathrm{~mm}$, and measure the mean activity along a sample diameter.

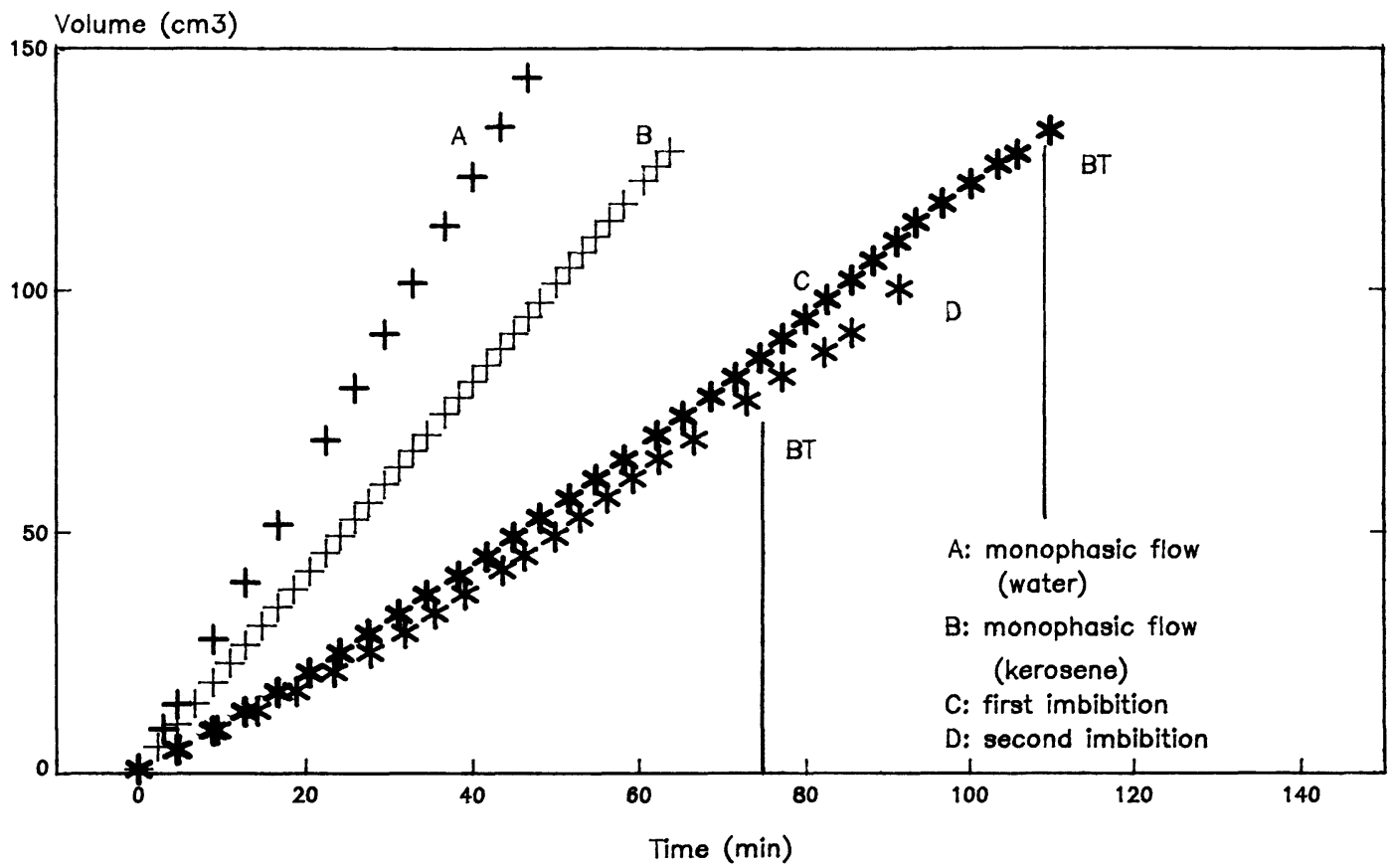

Fig. 1. - Accumulated volume vs. time for the packing of microspheres. - Pressure head $=4.8 \mathrm{~cm}$ of water.

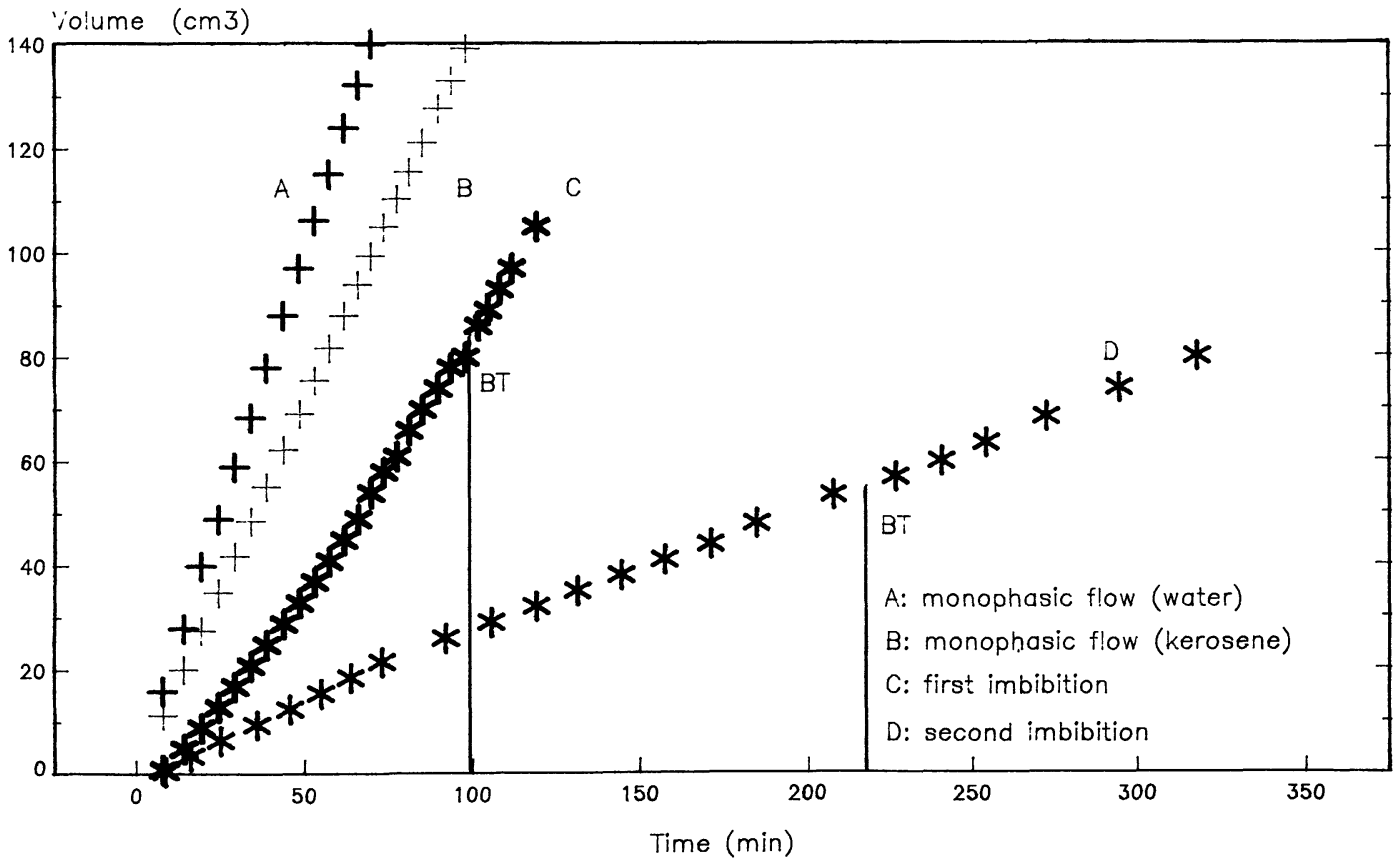

Fig. 2. - Accumulated volume vs. time for the Berea sandstone. - Pressure head $=41 \mathrm{~cm}$ of water. 


\section{Experimental results.}

We shall present in parallel the results obtained for both classes of porous media and consider successively the three phases of each experiment. We shall discuss the results in the next chapter.

3.1 FIRST IMBIBITION. - In a first experiment, we displace the kerosene, initially saturating the porous medium, by injecting tagged water under a constant pressure head.

The results at 4 different locations (the two extreme ones correspond to the inlet and outlet of the cylinders) are plotted in figures 3 and 4 for both types of samples. They show qualitatively the progressive replacement of kerosene by water as the front progresses. The activity is proportional to the

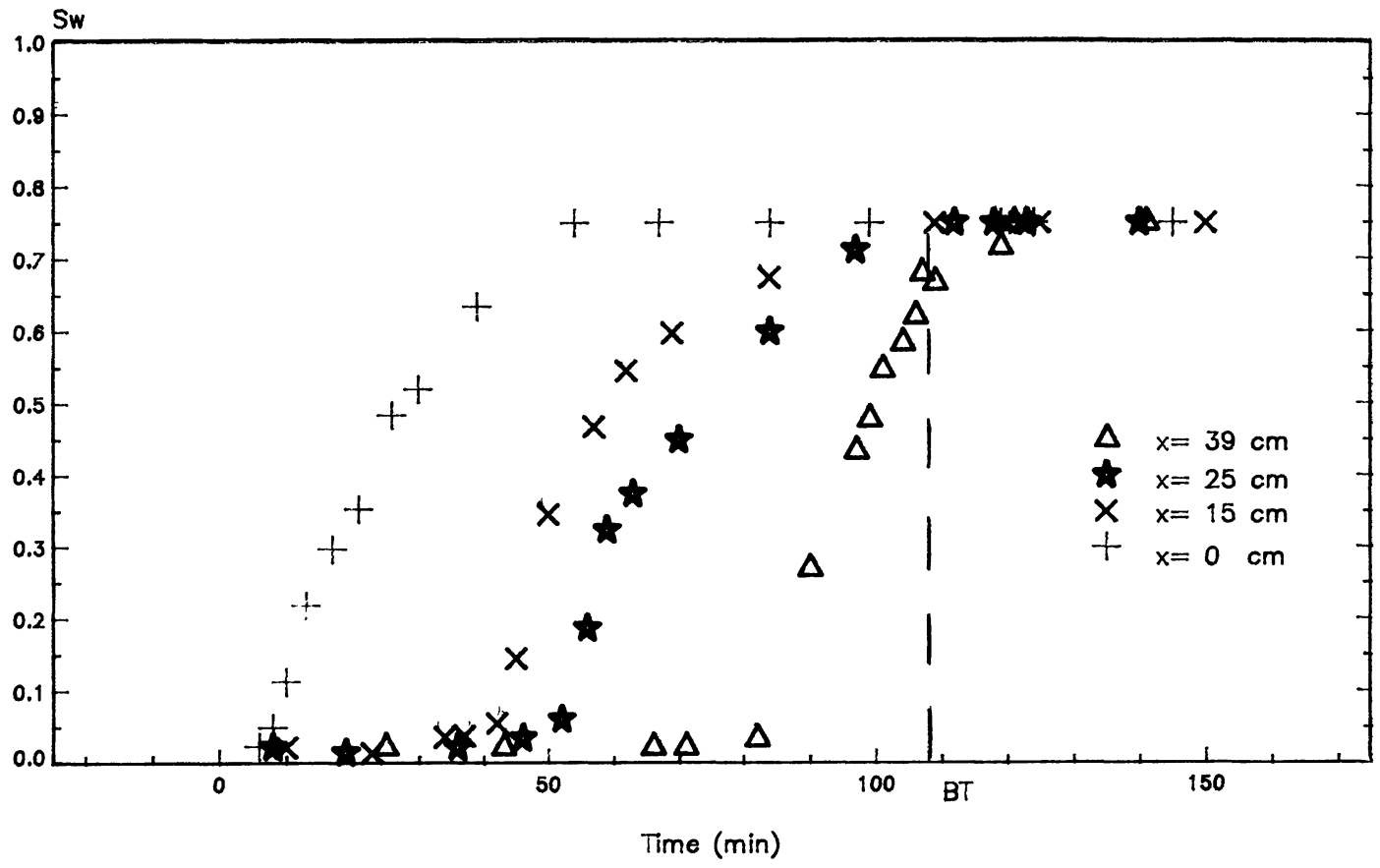

Fig. 3. - Tagged water saturation vs. time at four different locations for the packing of microspheres during a first imbibition. - Pressure head $=4.8 \mathrm{~cm}$ of water. Maximum $\mathrm{Ca}=1.5 \times 10^{-6}$.

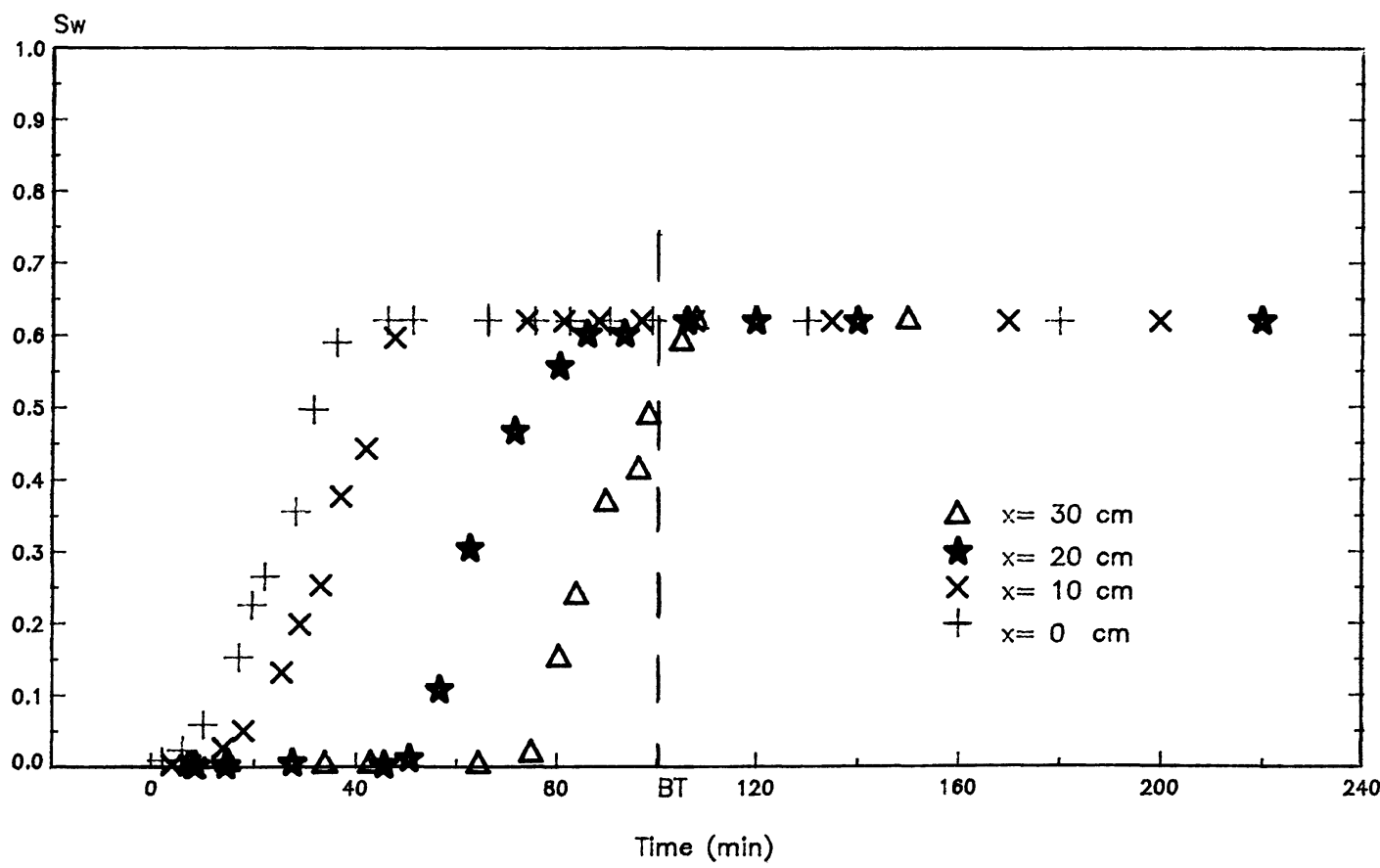

Fig. 4. - Tagged water saturation vs. time at four different locations for the Berea sandstone during a first imbibition. Pressure head $=41 \mathrm{~cm}$ of water. Maximum $\mathrm{Ca}=1.3 \times 10^{-6}$. 
amount of tagged water; thus the curves give a direct measurement of the tagged water saturation. In figures $1 \mathrm{C}$ and $2 \mathrm{C}$, we give the time variation of the injected volume corresponding to the same experiments. The flow rate is always smaller than that for monophasic water or oil flows in the same conditions.

In figures 1 and $\dot{2}$, curve $C$ should be compared with $B$ at early times where mostly kerosene flows, and with $\mathrm{A}$ at late times (mostly water). We also note that the flow rate is not constant with time which is a manifestation of the changing geometry of the fluid phases.

An important feature of the experiment is the fact that we obtain only an outflow of oil up to a time which is known as breakthrough time (B.T.) where the first drop of water is recovered at the outlet. Coming back to curves 3 and 4 we see that the B.T. corresponds approximately to the time where the water saturation at the outlet face of the medium has nearly reached its maximum value. It does not correspond, as would have been expected, to the time when the leading front of water touches the outlet face. This result is a consequence of the larger affinity of water for the porous medium as compared to oil. It will be analysed in more detail in chapter 4 .

Similar results have been obtained by nuclear magnetic resonance imaging in Orsay [13].

3.2 Drainage. - The next step consists in draining the medium, by injecting kerosene at the inlet of the sample, where the oil is initially at a constant residual saturation value $S_{\text {or }}$.

The experiment is performed by first using a small injection pressure which is kept constant until a certain residual concentration of water is reached all across the sample. Then, by successive steps, the pressure is increased until a prescribed low residual saturation of water $\left(S_{\text {wir }}\right)$ is reached. A typical value of $20 \%$ is obtained. In contrast to the imbibition phase, the injected fluid starts to exist as soon as it reaches the outlet face : indeed, some oil flows out of the sample as soon as the saturation of water close to the outlet starts to decrease. This result, too, will be discussed in chapter 4 .

3.3 SECOND IMBIBITION. - At this stage, a second imbibition is performed; tagged water is again injected at the inlet face under the same pressure and with the same activity as for the first imbibition. The water saturation is plotted in figures 5 and 6 as a function of time for the same locations as in the first experiment. The curves are qualitatively similar to those of figures 3 and 4 . The finite value of the base line corresponds to the residual saturation at the beginning of the second water injection. It is independent of the location of the probe, which shows that the drainage has left a uniform water saturation along the porous medium.

REVUE DE PHYSIQUE APPLIQUÉE. - T. 24, N 5, MAI 1989
The corresponding flow curves are plotted on figures $1 \mathrm{D}$ and $2 \mathrm{D}$. The flow rate values are lower than for the first imbibition and, also, than for the monophasic flows. In figure $2 \mathrm{D}$, we also note a decrease of the flow rate at large times in the more disordered Berea sample. Again, as in 3.1, we observe that breakthrough occurs only after the water saturation has reached its maximum value at the outlet face.

Let us come back to the water saturation data. An additional experiment has been carried on the Berea sample under conditions identical to those of figure 6 , except that the " connate » water which had been injected in the first imbibition was not tagged. Figure 7 displays the activity profile in this case. The difference between figures 6 and 7 gives the partial saturation variation for the residual water injected during the first imbibition as the second imbibition proceeds. It clearly shows that most (if not all) of this residual water is displaced in the process at the same rate as the kerosene. In particular we see that, at breakthrough, only a third of the water present at the outlet face is tagged water.

\section{Analysis of the results.}

4.1 COMPARISON WITH PREVIOUS WORKS. - The transport properties associated with the transient effects have been described in many works [14]. Of particular importance is the classical Buckley Leverett description which predicts the formation of a shock front for the distribution of concentration in an imbibition experiment. Recently, de Gennes [6] has added to this model the smearing effect of dispersive terms due to the convective exchange between the wetting and the non-wetting phase in the front region : this exchange can no larger take place when one of the phase becomes discontinuous. This effect was analysed by de Gennes [4] in the framework of the percolation theory. He predicted an hypodiffusive spreading of the imbibition invasion front characterized by the absence of « tails » in the saturation variation profiles.

On the other hand, he also suggested that these results might be modified by the presence of a surface film of the wetting phase on the pore walls. This effect of the coupling introduced by the film flow was taken into account by Bacri and Salin [15] to explain their experimental results. In their work, an acoustic transmission technique was used which (as in our case) integrates the concentration along the beam. They also perform a constant flow rate instead of a constant pressure invasion.

Their saturation profiles display clearly an hyperdiffusive behavior characterized by the fact that the time recordings (corresponding to our curves 5 and 6) depart from zero at the same time irrespective of the location of the probe along the flow axis. This 


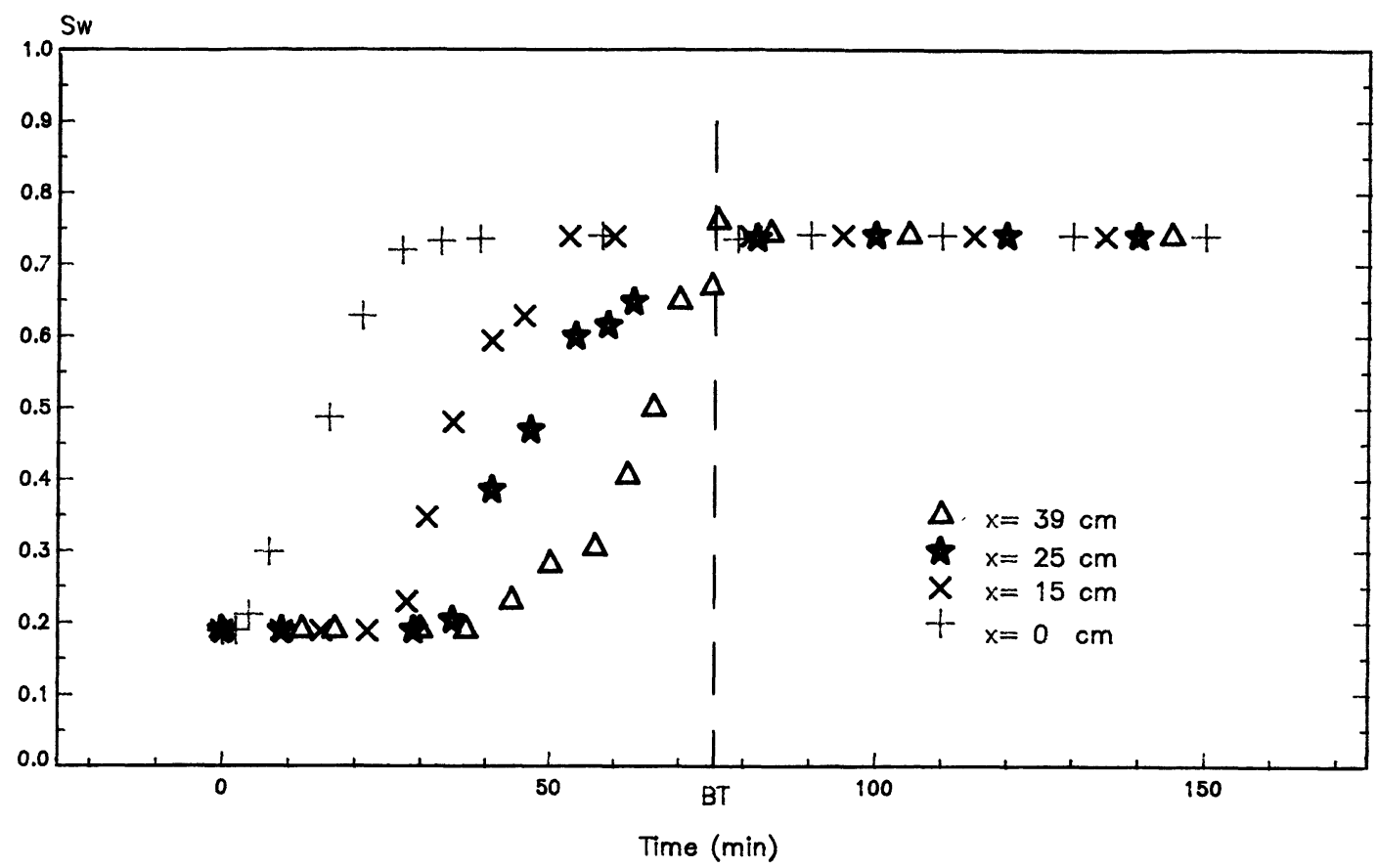

Fig. 5. - Tagged water saturation $v s$. time at four different locations for the packing of microscopheres during a second imbibition. - Pressure head $=4.8 \mathrm{~cm}$ of water. Maximum $\mathrm{Ca}=1.6 \times 10^{-6}$.

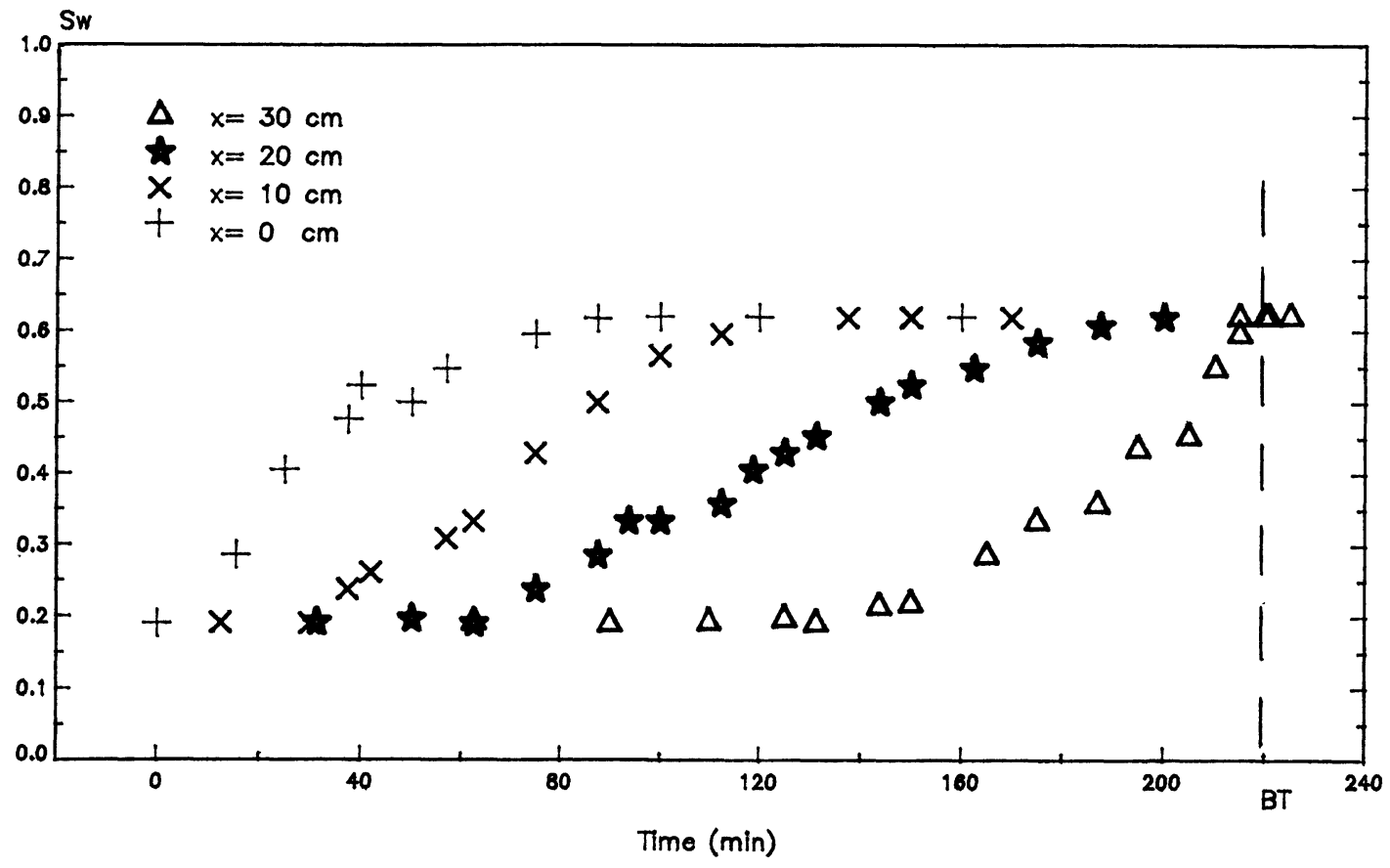

Fig. 6. - Tagged water saturation vs. time at four different locations for the Berea sandstone during a second imbibition. - Pressure head $=41 \mathrm{~cm}$ of water. Maximum $\mathrm{Ca}=3.5 \times 10^{-7}$.

result is consistent with the notion that, as a result of the second water injection, the water already present in the sample is mobilized immediately at different locations along the sample. We therefore think that the results of [15] are dominated by wall films effects while our observations correspond more closely to the predictions of [4].
We believe that this disagreement is due to the different initial treatment of the surface walls of the porous media. In the experiment mentioned above the material is initially filled with water (instead of kerosene in the present work) ; therefore, the water film remains in contact with the walls through all the sequence of drainages and imbibitions. In our work, 


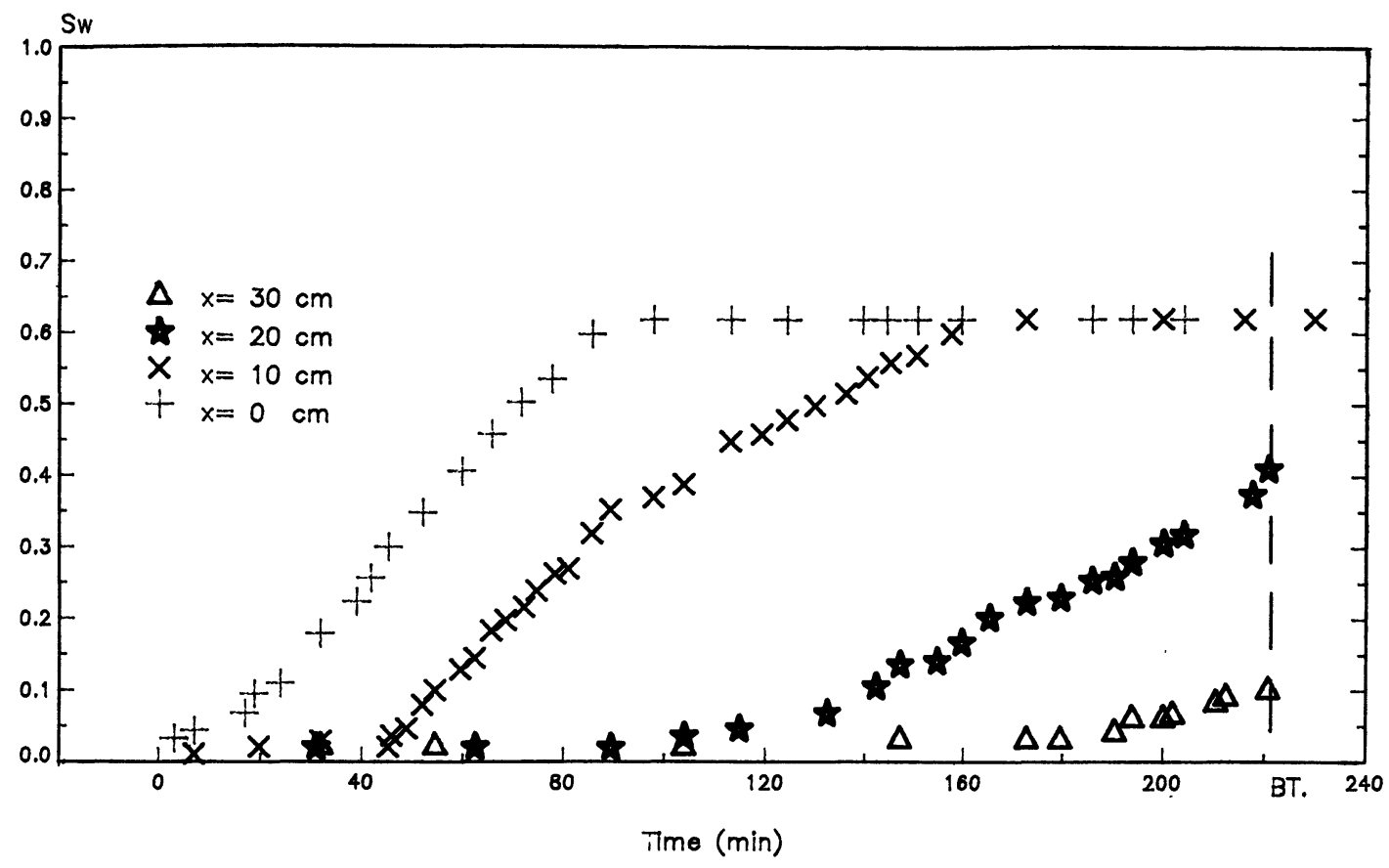

Fig. 7. - Tagged water saturation vs. time at four different locations for the Berea sandstone during a second imbibition with non tagged irreducible water. - Pressure head $=41 \mathrm{~cm}$ of water. Maximum $\mathrm{Ca}=3.5 \times 10^{-7}$.

a wetting film is not likely to have been present after we have replaced kerosene with water in the first imbibition. It is indeed a well known experimental fact that the surface properties are often controlled by the first liquid in contact with the solid surfaces. In oil engineering, for instance, the modification of the original wetting properties of rocks is an important step in improving assisted oil recovery. Let us note in particular the work of Morrow [10] who observed that crude oil and pure hydrocarbons may induce very different wettability modifications.

The sensitivity of our experiment would not be large enough to distinguish between a diffusive regime, and an hypodiffusive one (which would just be marked by a reduced «tail» at large times). However we clearly see that the initial breaks of the curves of figures 5 and 6 are positioned at different times according to the locations of the nuclear detector, as expected for the usual propagation of a concentration step such as predicted in [4].

On the other hand, let us note that, in the case of the high porosity of microspheres, the variation of concentration as well as the flow rate curves are fairly similar in the first and second imbibition. There is a much larger difference in the case of the more intricate geometry of the Berea sandstone, of lower porosity. However the variation corresponds to lower flow rates and a poorer transmission along the sample. This could be due to water trapped and not mobilizable from the first imbibition. It can also be due to the largest contrast in wettability between water and kerosene in quartz (sandstone) compared to amorphous glass beads.

Finally, we do not think that the use of constant pressure or flow rate injection processes plays any role in the differences of saturation profiles noted above. Indeed, during our experiments, the flow rate varies very slowly and by a factor of no more than 1.2. During a short time, therefore, we are close to a constant flow rate pattern and, since the saturation profile does not vary very fast with the velocity, the final fluid distribution will be the same.

4.2 A MODEL EXPERIMENT. - We want now to understand a number of additional features in our experiments, in particular, the different characteristics of the breakthrough transitions in the drainage and imbibition phases of the invasion, as well as the influence of the capillary forces on the flow rate values. For this purpose, we have developed a model experiment using parallel capillary tubes of different radii which simulate the effect of the pore size distribution. This approach extends a similar work by Chatzis and Dullien [16]. The geometry of the experimental set-up is given in figure 8 . We compare properties of the flow in two horizontal parallel glass tubes wetted by water (w). The outlet ends of the tubes are directly connected to the atmospheric pressure $p_{0}$. The inlets are both at a pressure $p_{2}$ and are connected through a manifold to a third capillary-tube also horizontal. This tube, in turn, is connected to a vessel where the fluid level is at a height $h$ above the outlets. 


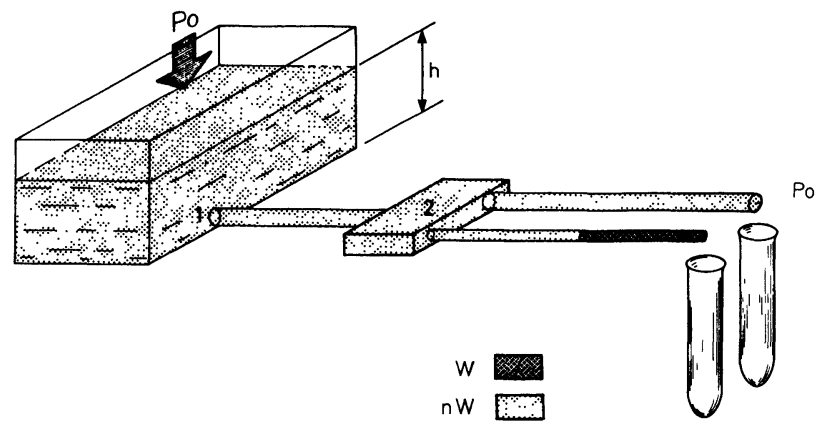

Fig. 8. - Sketch of the experimental device. $p_{2}=p_{\mathrm{o}}+$ $\rho g h-\Delta p_{12}$, where $\Delta p_{12}$ is the pressure head loss between points 1 and 2 .

The tubes are initially filled with water. In order to simulate the drainage part of the invasion, we inject, under a constant low driving pressure $p_{2}-p_{0}$, a less wetting fluid (kerosene).

At first, the meniscus moves much faster in the larger tube since both the capillary pressure opposing the flow and the viscous pressure drop at a given velocity are lower. When this meniscus has reached the end of the larger tube, it disappears and so does the corresponding capillary pressure opposing the flow in that tube. This results in an increase of the flow rate in the common branch and in the larger tube. The pressure drop $p_{1}-p_{2}$ in the two tubes increases and $p_{2}-p_{\mathrm{o}}$ correspondingly decreases. Very often, $p_{2}-p_{\mathrm{o}}$ is not big enough to balance the capillary forces in the smaller tube and the meniscus motion stops in that tube. This results explains why in the drainage of our real porous media, oil starts to flow out of the sample as soon as it reaches the outlet and the water displacement stops.

The situation is completely different in imbibition since, in that case, the effects of the capillary and viscous forces are antagonist.

In order to analyse these effects we have performed an experiment with tubes of equal diameters. One meniscus was artificially forced to lag behind the other; when the forward meniscus reaches the end of the capillary tube it gets pinned at its extremity. Then it distorts in such a way as to balance the applied pressure by a capillary component : it then presents a convex face to the outside atmosphere. If the applied pressure is low enough, this is a stable situation and the flow stops in the tube. The pressure loss $p_{1}-p_{2}$ in the common branch is subsequently reduced, and the flow rate in the other parallel tube increases. The process goes on up to the time when the second meniscus reaches the outlet. Then the wetting phase starts to flow out of both tubes if the applied pressure is large enough to overcome the maximum capillary pressure that can be developed on the pinned menisci.

In the case of tubes of different radii, one can also reach the outlet in all tubes without having any outflow of the wetting phase. Let us assume that, during the process, we reach a stage where all menisci get trapped and flow stops. Then, there is no viscous pressure loss and the full pressure is applied to the menisci ; this induces an outflow starting from the largest capillaries where menisci are less stable.

Hence the model experiment explains why, in the porous media, if the applied pressure is slightly above that in the largest pores, the oil starts to flow out only at the end of the process of motion of menisci towards the outlet face. At this time, the oil has reached its residual saturation and no new connected paths of oil gets established across the outlet part of the medium.

This residual saturation value is a collective property which results from an average over many pores. The above model explains how the information is transmitted between adjacent channels [11] through the variations of the pressure field transverse to the average flow rate.

We also believe that the effect of trapped menisci is the dominant factor to explain why, in our experiments in porous media, the flow rate is always smaller in two phase flows that in single phase ones. In the second imbibition in Berea sandstone, there are probably more pinned interfaces because of the more tortuous and less porous geometry. The flow rate is therefore markedly decreased with respect to the first imbibition.

\section{Conclusions.}

The experimental work reported above is a contribution to the important problems of diphasic flows in porous media.

One original feature of our work is the initial saturation of the sample with a non wetting fluid. This reduces the influence of wall films and gives normal diffusive characteristics for the invasion fronts instead of the strongly hyperdiffusive behaviour observed by other authors.

We also observe that the fluid injected during the imbibition starts to flow out only after the irreducible oil saturation is reached at the outlet. This is explained by the interplay between the capillary forces when the menisci reach the outlet and the viscous pressure drops inside the sample.

Another characteristic of the present experiment is the use of a constant driving pressure with an order of magnitude comparable to the capillary pressure : this technique allows us to monitor very easily both the pressure and the flow rate. In addition, it is possible to analyse with such a set up the threshold pressures values balancing the capillary forces in the zero flow limit.

We have realized a model experiment using capillary tubes which has been very useful for the analysis of these processes. It is presently developed in our 
laboratories in a more quantitative way in connection with detailed studies of the shape and dynamics of menisci in tubes.

For real porous media, we have proved that the nuclear tracer technique which we have used is a versatile approach for characterizing the distribution of the tagged phase flowing in a porous medium. This technique has also allowed us to finely analyse residual water displacement in a sequence of two imbibitions separated by a drainage phase.

In addition to the application to two phase flows which we discussed here, radioactive tracers can also be used to study dispersion and trapping.

The use of markers miscible in both phases would provide an additional information on the interfacial area in different injection conditions (in particular using different viscosity ratios between the two phases). More generally, the nuclear technique appears as a very general and flexible tool to study transport in polyphasic flows.

\section{Acknowledgments.}

We thank J. P. Hulin for numerous discussions during this work and for a detailed analysis of the manuscript. We also thank Geneviève Guillot for the complementary experiments made in Orsay on nuclear imaging.

We have had useful discussions with J. C. Bacri, D. Salin and R. Lenormand as well as a critical reading of the manuscript.

We also acknowledge the French Argentina cooperation agreement which has made possible the exchange between scientists during part of the time in which this project was carried.

\section{References}

[1] Bear J., Dynamics of Fluids in Porous Media, (American Elsevier) 1967.

[2] Anderson W. G., J. Pet ; Technol. (1986) P. 1605, (1987) 1453.

[3] Buckley S. E. and Leverett M. C., Trans. AIME (1942) p. 107.

[4] De Gennes P. G., Phys. Chem. Hydr. 4 (1983) 175 and [5].

[5] Lenormand R. and Zarcone C., Soc. Pet. Eng. J. 13264 (1984).

[6] DE Gennes P. G., in Physics of disordered materials, Eds. D. Adler, H. Fritzsche and S. R. Ovshinsky (Plenum Publishing Corporation) 1985, p. 227.

[7] Wilkinson D., Phys. Rev. A 30 (1984) 520.

[8] Lenormand R., C. R. Acad. Sci. (1980) and [7].

[9] LENORMAND R., private communication.

[10] Morrow N. R., Chem. Eng. Sci. 25 (1970) 1799 ; Morrow N. R., Lim H. and WArd J. S., Soc. Pet. Eng. J. (Feb. 1986) p. 89.
[11] Sydansk R. D., Soc. Pet. Eng. J. (1980) p. 1329.

[12] Grattoni C., Rosen M., Chertcoff R. and Bidner M., Chem. Eng. Sci. 42 (1987) 2055.

[13] Guillot G. and Rosen M., Unpublished.

[14] Dullien F. A. L., Porous Media Fluid Transport and Pore Structure (Academic Press) 1979, Chapter 6.

[15] BACRi J. C. and SAlin D., J. Phys. Lett. France 46 (1985) L 467. In the reference they quote (p. 470) that « ... the dry sample is first saturated in oil with a vacuum impregnation technique. » corresponding to the procedure applied here. This is apparently a misprint as was indicated recently to us by these authors who told us that, in fact, the hyperdiffusion regime was only obtained if the dry sample had been first saturated in water.

[16] Chatzis I., Thesis, Univ. fo Waterloo, Canada (1978). 